(REVIEW ARTICLE)

\title{
Some considerations on care of the elderly, using evolutionary genetic models
}

\author{
Takahiro Miyo* \\ Association for Evolutionary Research on Aging Populations, 2-17-4 Misumi-cho, Higashimurayama-shi, Tokyo 189-0023, \\ Japan.
}

Publication history: Received on 06 November 2020; revised on 14 November 2020; accepted on 17 November 2020

Article DOI: https://doi.org/10.30574/wjarr.2020.8.2.0414

\begin{abstract}
Some evolutionary genetic models that assume pleiotropic constraints among care for old parents and care for infants are developed, in order to examine the evolution of care for the elderly within a population, in which population aging coupled with a tendency toward a decrease in population size is in progress. Not only does this study suggest that high levels of care for old parents could evolve within the population if the cost of care of the elderly is not too heavy, but it also suggests that, if younger generations experience a high cost of elderly care, this behavior might be eliminated from the population, resulting in the loss of high levels of care for infants. It is also suggested that the benefit of the help from the elderly is essential, not only for the behavior of care for old parents to be maintained within the population, but also for population growth, even though there may be a high cost of elderly care. Based on the results obtained, some social issues that some countries, such as Japan, are now, or will be, faced with are discussed.
\end{abstract}

Keywords: Elderly care; Evolutionary genetic models; Grandparents; Parent-offspring interactions; Pleiotropic constraints.

\section{Introduction}

In the previous paper [1], I have raised the question of why we care for old parents, because old parents have already ceased reproduction, so that care for old parents should provide no inclusive fitness benefits [2-4] for us. At first sight, therefore, the fact that most people, as far as I know, do indeed care for their old parents seems to be a kind of paradox, and mechanisms underlying elderly care need to be explained in the light of evolution. Using some evolutionary genetic models, which assume pleiotropic constraints between parent-offspring interactions, I examined the evolution of elderly care, and suggested that establishing solid bonds with parents during infancy could be selectively favorable, even though we have to care for old parents at a heavy cost in the course of future life [1].

There is a worldwide trend toward an increase in the percentage of the elderly, and Japan has the highest in the world [5]. The number of people who need long-term care is increasing among the elderly, and the number of the elderly with dementia is anticipated to keep increasing gradually in the future [5]. Although the tendency toward an increase in the percentage of the elderly is seen worldwide, the problems that such aging countries as Japan are now or will be faced with seem more difficult to deal with, because the population is not only aging but also decreasing. In general, the relationship between changes in fecundity and corresponding changes in the intrinsic rate of increase tends to decrease with age, if the population is increasing (p. 192 of Charlesworth [6]). However, under the conditions of population aging and diminishing population size, the relationship between changes in fecundity and corresponding changes in the intrinsic rate of increase may increase with age, in spite of the fact that elderly people may have ceased reproduction (Figure 3 of Miyo [1]). Therefore, care of the elderly, which involves a lot of cost, may become an increasingly serious problem as the tendency toward a decrease in population size strengthens.

\footnotetext{
${ }^{*}$ Corresponding author: Takahiro Miyo

Association for Evolutionary Research on Aging Populations, 2-17-4 Misumi-cho, Higashimurayama-shi, Tokyo 189-0023, Japan.. 
The long lifespan after cessation of reproduction in humans and some mammals has been one of the mysteries in evolutionary biology $[7,8]$. One of the hypotheses, the so-called grandmother hypothesis, maintains that the long lifespan after cessation of reproduction may have some selectively advantageous value, because the elderly could then make direct and indirect contributions to reproductive success of their younger kin and close relatives, so that they could receive inclusive fitness benefits through helping younger relatives [9, 10,11]. According to the grandmother hypothesis, the long lifespan after cessation of reproduction of the elderly should actually provide some benefits in fitness; in fact, it was shown that women with a prolonged post-reproductive lifespan have greater fitness in some premodern populations, by means of enhancing the lifetime reproductive success of their offspring [7, 12]. However, the benefits from the prolonged post-reproductive lifespan do not last forever, but wane off as the age of the elderly increases and their health decreases [13]. Therefore, it is desirable to discuss the problems of care of the elderly, taking into account not only the cost of elderly care but also the benefit that the elderly could produce through their help toward younger relatives. This is especially necessary for aging and diminishing countries such as Japan, because positive as well as negative contributions from elderly people would be imposed heavily on us as their aging proceeds (Figure 3 of Miyo [1]). In order to do this, we have to take into account the effects of interactions among generations, from grandparents to grandchildren.

In this study, I have examined elderly care from the standpoint of evolutionary genetics, using some models which take into account not only parent-offspring interactions but also the benefits and costs from interactions between grandparents and grandchildren. Based on the results obtained, some issues which decreasing and aging populations such as Japan are now, or will be, faced with are discussed.

\section{Some Models of the Evolution of Care of the Elderly}

\subsection{Discrete-Generation Model Focusing on Parent-Offspring Interactions}

\subsubsection{General Considerations}

In the previous paper, I used some evolutionary genetic models, assuming pleiotropic constraints between care for infants by parents and care for old parents by their children [1]. In general, it is widely believed that most behaviors are complex and are affected by multiple factors [14]. Therefore, it is natural that behaviors among family members should vary in relation to social and physical conditions and ecological circumstances [15], but there is little doubt that establishing a solid relationship with parents has substantial selective advantages especially for infants, because we cannot survive without care from others (usually parents) during infancy $[16,17]$. Referring to the evidence from some utopian social experiments including a kibbutz in Israel, Arnhart [18] suggested that prohibiting the parent-offspring bond would result in unbearable frustration for most people, and that this bond must take its place in the organization of human desires. Therefore, I assume that establishing a solid parent-offspring bond is part of human nature, and that care of the elderly should be an inevitable consequence of the substantial selective advantages of the parent-offspring bond for infants (a pleiotropic constraint) [1].

\subsubsection{Discrete-Generation Model (Type I)}

In the previous paper [1], I reported results using the age-structured version of this model. Although discretegeneration models cannot take into account the age-specificities of life events in detail, I would like to introduce this model here, in order to make clearer the models including effects from grandparents as well as grandchildren described in the following sections.

Using a Type I discrete-generation model, the conditions for the spread of an allele $(A)$ causing high levels of care for the elderly as well as infants are examined when it is at a low frequency within an infinite, random mating population with discrete generations. In the initial condition, the majority of the population members are assumed to have a relatively indifferent allele $a$. $A$ is assumed to be inherited as an autosomal dominant factor and is expressed in males and females to the same extent. Because this model focuses on parent-offspring interactions, and because the inclusive fitnesses of genotypes may not be affected after cessation of reproduction, the fitnesses of genotypes are assumed to be determined by the genotypes of their parents, and the cost of elderly care imposed on offspring to be incorporated into selection coefficients $(s)$. Therefore, even though individuals have the same genotype, their fitnesses could be different among families. For example, the fitness of $A a$ offspring with $A A$ and $a a$ parents may be different from that of $A a$ offspring with $A a$ and $A a$ parents. Table 1 summarizes this model briefly. 
Table 1 The discrete-generation model of elderly care (Type I).

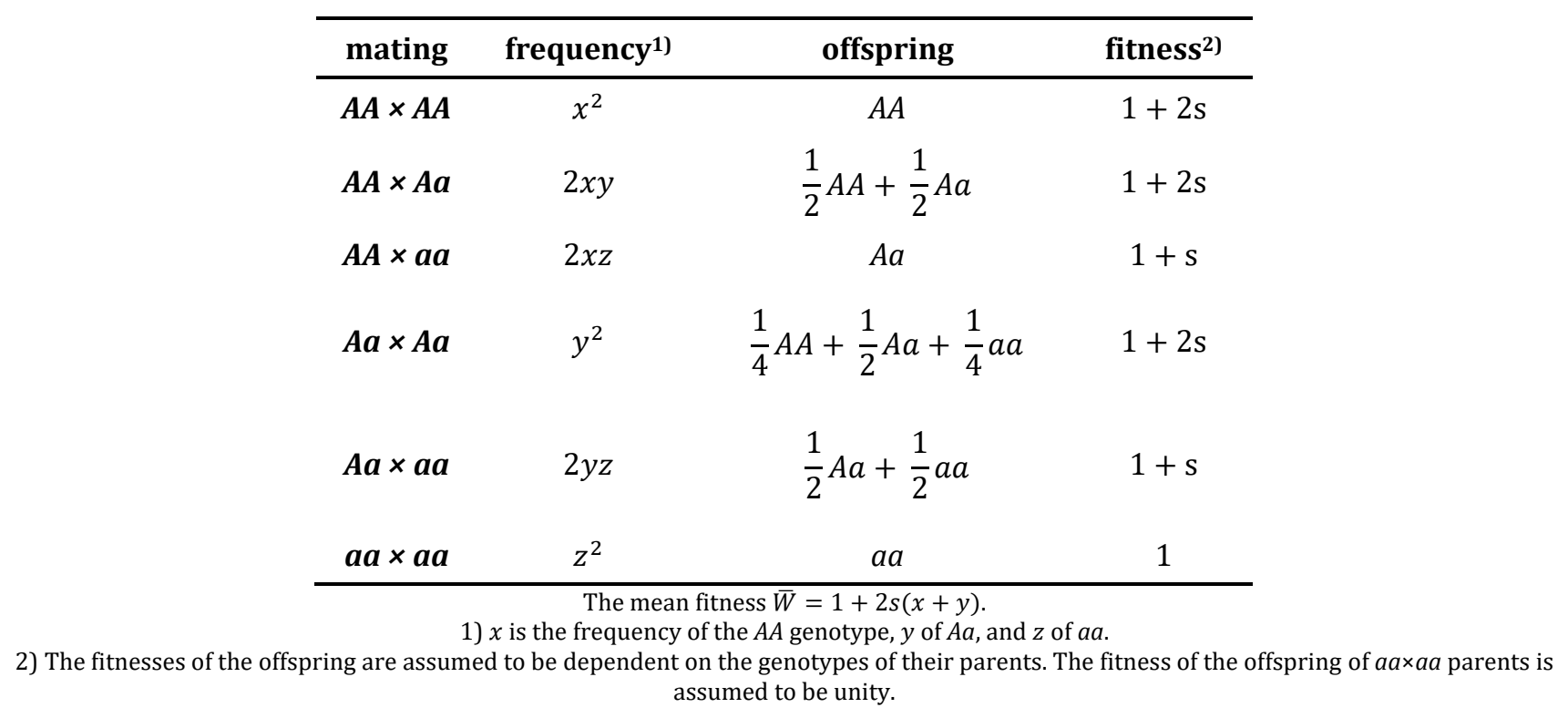

\subsubsection{Spread of a High-Care Gene}

In the previous study, the conditions for the initial increase in the frequency of $A$ allele causing high levels of care for the elderly as well as infants were examined by using some evolutionary genetic models taking age-structures into account [1]. When the $A$ frequency becomes higher in the population, so that the allele $A$ is not rare any more, various combinations of genotypes among parents and family members would be expected. In such situations, interactions between parents and offspring would be much more complex than the initial condition where $A$ allele is still rare, and the simplified discrete-generation model (Table 1) is necessary here.

Following some basic algebraic procedures in population genetics (cf. [19]), the frequencies of $A A, A a$, and $a a$ in the next generation, $x^{\prime}, y^{\prime}$ and $z^{\prime}$, respectively, are

$$
\begin{aligned}
& x^{\prime}=p^{2}(1+2 s) / \bar{W} \\
& y^{\prime}=p[y(1+2 s)+2 z(1+s)] / \bar{W} \\
& z^{\prime}=q(q+s y) / \bar{W}
\end{aligned}
$$

where the mean fitness of the population $\bar{W}=1+2 s(x+y)$, the frequency of $A$ allele $p=x+y / 2$, and the frequency of $a$ allele $q=z+y / 2$. The frequency of $A$ allele in the next generation $p^{\prime}=x^{\prime}+y^{\prime} / 2$, so that the expression for the change in the frequency of $A$ allele, $\Delta p$, is obtained as

$$
\Delta p=s p z / \bar{W}
$$

Because $0 \leq p \leq 1$ and $0 \leq z \leq 1$, the allele $A$ would always increase in frequency under polymorphic conditions, if the selection coefficient $s$ is positive. $s$ is set, based on the difference in the intrinsic rate of natural increase (hereafter denoted by $r$ ) between $A a$ and $a a$ genotypes (the assumed initial condition), so that the allele $A$ would increase in frequency, if $A a$ has a higher $r$ than $a a$. Therefore, the initial spread of the $A$ allele is consistent with the increase in frequency of the $A$ allele during the period when it is not rare any more. On the other hand, if there is no difference in fitness between genotypes $(s=0)$, the population should maintain the initial conditions. In this model, these conditions would result from the situations, where care of the elderly imposes the fecundity cost on $A a$ genotype, resulting in $r$ equivalent to that of the relatively indifferent genotype (Table 1 of Miyo [1]). Furthermore, the high care $A$ allele would be excluded from the population, if $a a$ has a higher $r$ than $A a$, resulting in a negative $s$ value. These results are consistent with the results reported in Miyo [1]. Details of the results are available therein. 


\subsection{Age-Structured Model Involving the Help and Cost from Grandparents and Grandchildren}

\subsubsection{General Considerations}

Taking age-structure into account is essential for discussing the evolution of care of the elderly, not only because it may allow us to incorporate age-specificities of life events during infancy and late life into models, but also because it allows us to consider whether the population is increasing or decreasing. In discrete-generation models, we are concerned with relative frequencies of genotypes and genes, but we cannot pay attention to whether the population is increasing or decreasing. Since the consequences of elderly care when the population is decreasing might be totally different from those when the population is increasing [1], models taking age-structure into account are desirable for discussing the evolution of care of the elderly, although they are inevitably more complex.

The models described so far focused on parent-offspring interactions ([1] and the aforementioned Type I discretegeneration model), in which care for infants by parents (positive fitness effects on offspring and therefore on parents) and care for the elderly by children (positive survival effect on parents but negative fecundity costs for children) are taken into account. Although we pay more attention to the cost of elderly care in everyday life, post-reproductive period individuals could confer indirect selective advantages via their contributions to the reproductive success of younger close relatives $[7,9,20,21]$. Therefore, not only parent-offspring interactions but also contributions from grandparents and grandchildren, which could be positive or negative depending on temporal circumstances, are included in the models below.

\subsubsection{Age-Structured Population Model}

I used the same vital statistics for the hypothetical population suffering from population aging and diminishing population size and the same age-structured population scheme as in the previous study [1], following the general theory of kin selection in age-structured populations [22]. In this study, furthermore, the effects from grandparents as well as grandchildren are taken into account, in addition to parent-offspring interactions.

In the initial condition, the genotype carrying the high care $A$ allele is $A a$, and its mates are $a a$, because homozygous individuals carrying two $A$ alleles and mating betweens two $A a$ individuals are so rare that these combinations can be ignored. Under these conditions, $A a$ individuals are engaged only $A a \times a a$ matings, so that during the period when $A$ is rare, the family with the $A a$ genotype would be expected to produce the $A a$ genotype every generation with probability one-half. As a result, $A a$ individuals would be expected to have one $A a$ grandparent and one $A a$ parent. Therefore, in addition to the $A a$ parent-offspring interactions, $A a$ individuals may be expected to receive additional advantages for survival and/or reproduction from their $A a$ grandparent and $A a$ grandchildren, but at the same time a higher cost of elderly care may be imposed on their $A a$. Figure 1 summarizes the scheme of this age-structured population model.

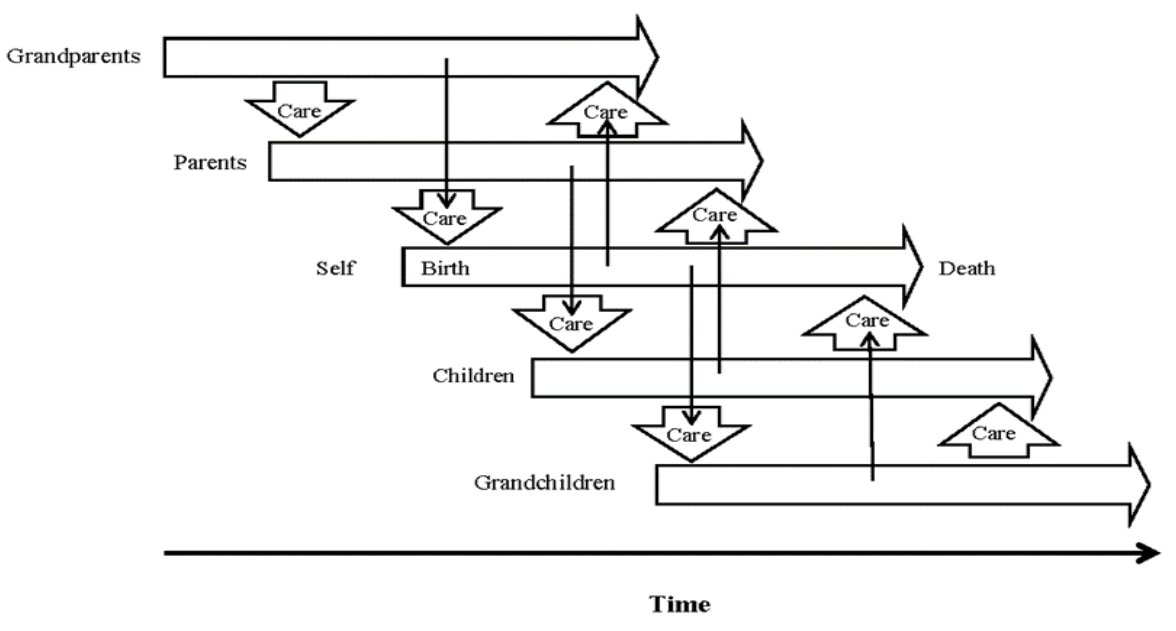

Figure 1 A schematic diagram depicting interactions among family members, including not only parents and offspring but also grandparents and grandchildren.

In this study, the vital statistics for the Aa genotype are the same as those of the high care genotype in Miyo [1]. These are for the hypothetical population suffering from population aging and decrease, which were created using the Japanese population statistics; based on these vital statistics, the intrinsic rate of natural increase, generation time and 
net reproduction rate in the 5-year time unit were estimated as $-0.081,6.56$ (about 32.8 years) and 0.59 , respectively (refer to [1] for more detailed information). During the initial phase, when $A$ is still rare, $A a$ offspring will receive high levels of care during infancy, because one of their parents is $A a$, while $A a$ parents will receive high levels of care late in life, because half of the offspring are expected to be $A a$. Therefore, through parent-offspring interactions, the $A a$ genotype would be expected to have higher survival rates during infancy than the aa genotype, as well as late in life. For the a genotype, vital statistics for the relatively indifferent genotype in Miyo [1] were used, in which the age-specific survival rates (hereafter denoted by $P(x)$ ), during age-classes 0-2 (birth to 9 years old) and age-classes 15-17 (70 to 84 years old) were assumed to be $5 \%$ lower than those of the high care genotype. In this case, the relative fitness of the high care genotype is estimated to be 1.17 , if the fitness of the relatively indifferent genotype is assumed to be unity, based on $r$ for the a genotype (The Euler-Lotka equation for the high care genotype, whose $r$ is replaced with $r$ for the aa genotype yields the relative fitness of the high care genotype; cf. equation (4.78) of Charlesworth [6]). Although no specific form of indifferent behavior is assumed here, child maltreatment and elderly maltreatment often result in tragic consequences, so that the difference in fitness between the two genotypes might be conservatively evaluated here.

In addition to the basic parent-offspring interactions, the $A a$ genotype is assumed to receive benefits for $P(0)$ and fecundity (hereafter denoted by $m(x)$ ) from care by grandparents, negative cost effects (costs) for $m(x)$ as a result of caring for grandparents, and benefits for $P(17)$ from aid from grandchildren (Figure 2). The costs and benefits for $m(x)$ are assumed to be the same at the all reproductive ages. The consequences of the benefits and costs were evaluated with several values, and represented by multipliers, such as $P(0) \cdot(1+g)$ and $m(x) \cdot(1+g)(1-c)$, where $g$ represents the effect of the help from grandparents and $c$ the cost of elderly care. Because $P(0)$ could become $>1$, if the benefit $g$ for $P(0)$ is too large, $g$ for $P(0)$ is capped at 0.03 when $g$ values are more than 0.05 . The effects of the cost of elderly care and the benefit of the help from the elderly on $r$, the generation time $(T)$, the net reproduction rate $(R)$, and the total fertility rate ( $F$ : the sum of the age-specific fecundities over all reproductive ages) are summarized in Table 2.
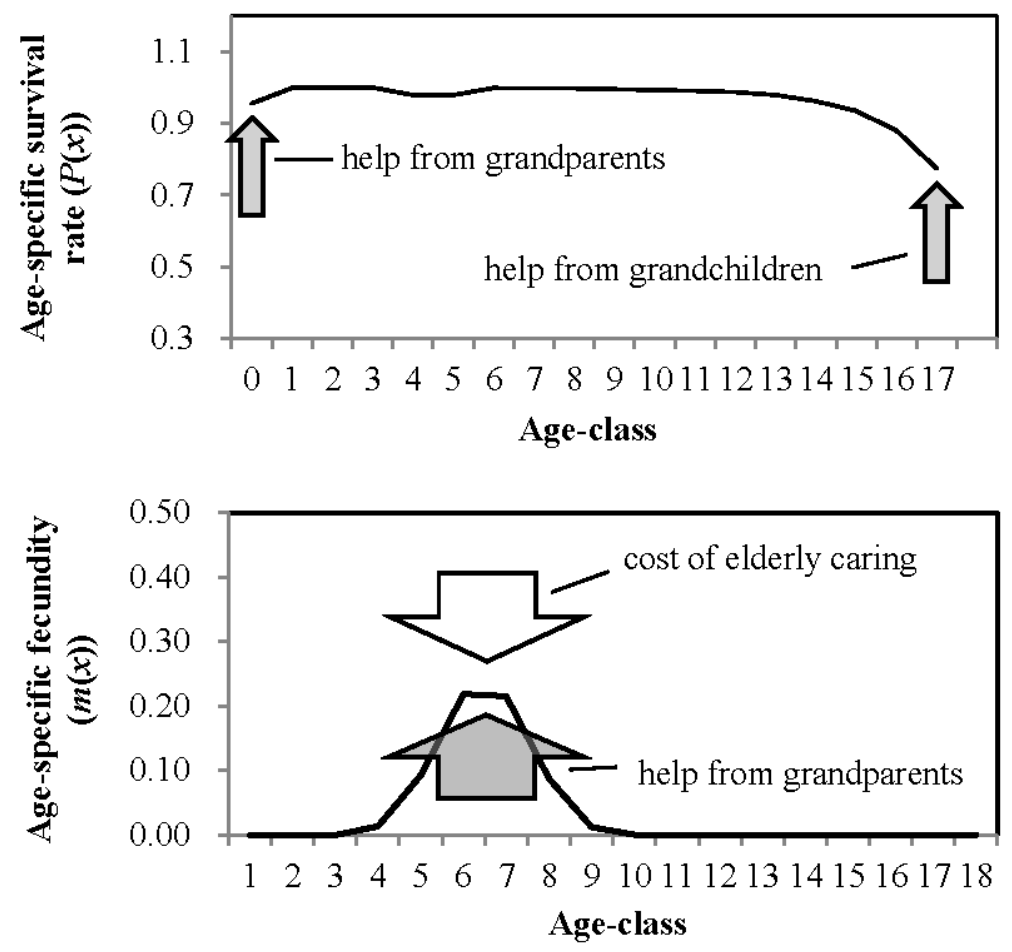

Figure 2 The effects of the benefit of the help from grandparents and grandchildren and the cost of elderly care on demographic parameters of the high care $A a$ genotype. 
Table 2 Demographic effects of the cost and benefit of family interactions.

\begin{tabular}{|c|c|c|c|c|c|}
\hline cost & help & $r^{1)}$ & $T^{2)}$ & $R^{3)}$ & $F^{4)}$ \\
\hline \multicolumn{2}{|c|}{ original $A a$} & -0.08102 & 6.55874 & 0.58985 & 0.63992 \\
\hline \multirow{7}{*}{0.10} & 0.00 & -0.09706 & 6.57584 & 0.53087 & 0.57593 \\
\hline & 0.01 & -0.09403 & 6.57262 & 0.54154 & 0.58169 \\
\hline & 0.02 & -0.09104 & 6.56942 & 0.55232 & 0.58745 \\
\hline & 0.05 & -0.08514 & 6.56314 & 0.57413 & 0.60473 \\
\hline & 0.10 & -0.07804 & 6.55558 & 0.60147 & 0.63352 \\
\hline & 0.15 & -0.07126 & 6.54859 & 0.62881 & 0.66232 \\
\hline & 0.20 & -0.06476 & 6.54152 & 0.65615 & 0.69112 \\
\hline \multirow{7}{*}{0.15} & 0.00 & -0.10575 & 6.58511 & 0.50138 & 0.54393 \\
\hline & 0.01 & -0.10272 & 6.58188 & 0.51145 & 0.54937 \\
\hline & 0.02 & -0.09973 & 6.57869 & 0.52163 & 0.55481 \\
\hline & 0.05 & -0.09384 & 6.57241 & 0.54224 & 0.57113 \\
\hline & 0.10 & -0.08676 & 6.56486 & 0.56806 & 0.59833 \\
\hline & 0.15 & -0.07998 & 6.55764 & 0.59388 & 0.62552 \\
\hline & 0.20 & -0.07349 & 6.55104 & 0.61970 & 0.65272 \\
\hline \multirow{7}{*}{0.20} & 0.00 & -0.11495 & 6.59491 & 0.47188 & 0.51194 \\
\hline & 0.01 & -0.11193 & 6.59169 & 0.48137 & 0.51706 \\
\hline & 0.02 & -0.10894 & 6.58850 & 0.49095 & 0.52218 \\
\hline & 0.05 & -0.10306 & 6.58223 & 0.51034 & 0.53753 \\
\hline & 0.10 & -0.09598 & 6.57470 & 0.53464 & 0.56313 \\
\hline & 0.15 & -0.08922 & 6.56749 & 0.55895 & 0.58873 \\
\hline & 0.20 & -0.08274 & 6.56058 & 0.58325 & 0.61432 \\
\hline
\end{tabular}

Broken lines in the table indicate the thresholds in help which result in superior values than the original $A a$. 1) the intrinsic rate of increase. ${ }^{2)}$ the generation time. ${ }^{3)}$ the net reproduction rate. ${ }^{4)}$ the total fertility rate.

\subsubsection{Spread of a High-Care Gene}

Table 3 shows numerical values of the helping and cost effects for the high care genotype that result in the same $r$ as the relatively indifferent genotype. Under these circumstances, $A a$ and $a a$ would have the same $r$; this implies that, if values of the cost for the respective help effects get larger than the values listed in Table 3 , the $A$ allele would be eliminated from the initial population composed of the relatively indifferent genotype.

Table 3 The benefits and costs of care of the elderly, which result in an $r$ for $A a$ equivalent to that of $a a$, with the agestructured population model.

\begin{tabular}{cccccccc}
\hline Benefit & 0.00 & 0.01 & 0.02 & 0.05 & 0.10 & 0.15 & 0.20 \\
\hline cost & 0.1426 & 0.1595 & 0.1759 & 0.2072 & 0.2433 & 0.2762 & 0.3063 \\
\hline
\end{tabular}

In the previous paper [1], the cost of elderly care was taken into account, but the benefit of the help from the elderly was not. Under these conditions, it was suggested that the behavior of care for old parents could evolve by establishing a solid relationship with parents during infancy. However, it was also suggested that if the cost of elderly care is too 
large, this behavior could be excluded from the population [1]. In this study, in addition to the parent-offspring interactions, the benefit of the help from the elderly is taken into account. The benefit may be expected from the potential selective advantage of the elderly during the long lifespan after cessation of reproduction, which is suggested by the grandmother hypothesis $[7,9,10]$.

It is assumed that the rare $A a$ individuals are introduced into a population fixed initially for the $a a$ genotype, of which frequencies are set to be 1:100 ( $A a: a a)$. After calculating the trajectories of the number of $A a$ and $a a$ newborns, the relative frequency of the $A$ allele $\left(p_{A}\right)$ is approximately estimated as follows (see [1] for more detailed information).

$$
p_{A}=\text { (the number of } A a \text { newborns) } /[2 \text { (the number of } a a \text { newborns) }]
$$

As with the previous study, if the cost of elderly care is too large, the fate of the behavior of care for old parents should be exclusion from the initial population. However, if the cost is not too large, the $A$ allele could invade the initial $a a$ population with a small amount help from the elderly (Figure 3a). Furthermore, it is suggested that, even when the cost of elderly care becomes larger, the $A$ allele could invade if a corresponding larger amount help is provided by the elderly (Figure 3b).
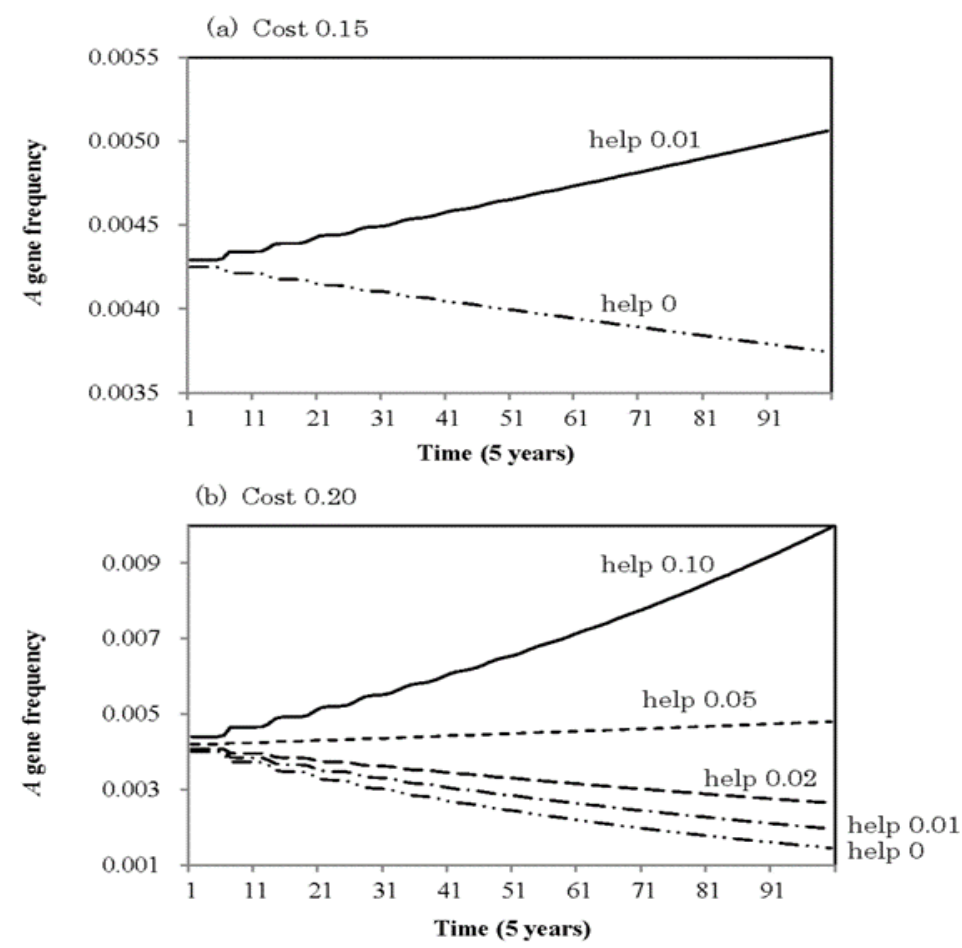

Figure 3 The effects of the help from grandparents and grandchildren in the cases (a) $c=0.15$ and (b) $c=0.20$.

Population aging coupled with a decrease in the number of children, which some countries such as Japan is now or will be faced with, could result in an enormous cost for younger generations (for example, it is anticipated that 1.3 persons of working age (aged 15 64) would have to support one elderly person (aged over 65) in 2065 [5]). If younger generations only receive an enormous cost but no benefit from the elderly, there will be selection against a predisposition toward high levels of care (as represented by the high care gene in this study). As a result of the exclusion of the high care gene, it is projected that not only the behavior of care for old parents, but also high levels of care for infants, would be ultimately be eliminated from the population (remember that care for old parents and care for infants are assumed to be pleiotropically constrained in this study). The resulting society might experience serious social conditions, which would be harsh not only to the elderly but also to infants. However, as summarized in Table 2 and Figure 3, it is possible that $r$ could be increased despite the cost of elderly care, if the benefit of the help from the elderly is sufficiently large. This suggests that the benefit of the help from the elderly could contribute to some extent to the rate of population growth. Therefore, it seems necessary to consider how we could derive as large help effects from the elderly as possible and how we could manage the enormous cost of elderly care as effectively as possible. 


\subsection{Discrete-Generation Model Involving Benefits and Costs from Grandparents and Grandchildren}

\subsubsection{General Considerations}

Because we are concerned with care of the elderly, which is in general an age-related, we should incorporate agestructure into the population genetic models. However, as mentioned above, after $A$ has increased sufficiently within the population, several different combinations of genotypes among mates and family members are produced, so it becomes very difficult to model their interactions. Here, I use another discrete-generation model (Type II), which takes into account effects from grandparents and grandchildren in addition to parent-offspring interactions. Because it is much more complex than the Type I discrete-generation model, I conducted some numerical simulations in order to obtain some insights into the influences of the potential cost and benefit from the elderly on the evolution of care of the elderly and also population growth. The scheme of the Type II model was derived in part from population genetic models in Charlesworth [23] and Charlesworth and Charlesworth [24].

\subsubsection{Discrete-Generation Model (Type II)}

In the Type I discrete-generation model, the fitnesses of individuals are assumed to be dependent only on the genotypes of parents. In the Type II model, the fitnesses of individuals are assumed to depend not only on genotypes of parents, but also on genotypes of grandparents. Figure 4 illustrates as an example the way to calculate the fitness of $A a$ born to $A a$ and $a a$ parents. Although it might be interesting to distinguish sex of individuals in considering interactions among family members, because fathers and mothers, and/or grandfathers and grandmothers may have different roles in interactions among family members $[25,26]$, this makes the models much more complicated, so that the presumed differences between the sexes in contribution to interactions are unfortunately ignored. Because the sexes are ignored, there are, for example, four combinations of parental genotypes that could produce $A a$ offspring, and there are much more combinations of grandparents that could produce parental genotypes. Taking into account genotypes of grandparents as well as parents, there are 10 family-types, and 55 combinations in total among them. The fitnesses of individuals are calculated for each of the 55 combinations of family-types. The fitnesses of individuals are increased or decreased, dependent on how many parents carry $A$ allele $(0 \sim 2$ parents) and how many grandparents carry $A$ allele $(0 \sim 4$ grandparents). It is assumed that offspring gain the benefit $p$ per one parent carrying $A$ allele, and the benefit $g$ per one grandparent carrying $A$ allele. At the same time, if there are any parents who carry the allele $A$ in the family, the parent(s) would receive the cost of high levels of care for his/her parents (grandparents), so that it is assumed that the cost $2 c$ is charged per one parent carrying $A$ allele. Therefore, in the case of the combination of the two family-types illustrated in Figure 4, the fitness of $A a$ and $a a$ born to that family is assigned to have a fitness value $(1+p+g)(1-$ $2 c$ ), because they are born to the family having one Aa parent and one Aa grandparent. On the other hand, the fitness of $a a$ individuals who are born to the pure $a a$ family is assumed to be unity (no benefit, but no cost).

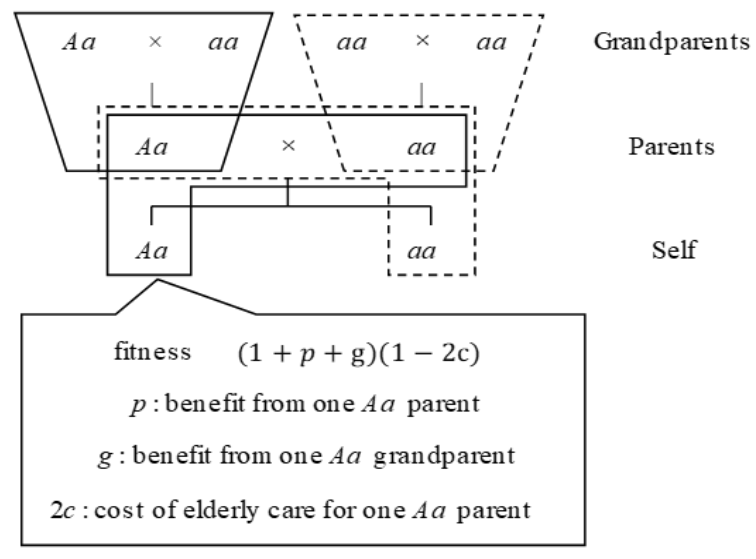

Figure 4 Illustration of how to calculate the fitness of $A a$ genotype born to one of the 55 family-types considered.

In the Type II discrete-generation model, individual fitnesses vary, depending on what genotypes their parents as well as their grandparents have, even though individuals have the same genotypes; therefore, in order to evaluate population genetic processes concerning care of the elderly, frequencies of family-types involving parents as well as offspring are calculated, rather than individual genotypic frequencies. (For example, the genotype $A a$ born to $A A$ and $a a$ parents is necessary to be distinguished from the genotype $A a$ born to $A a$ and $A a$ parents.) In every generation, relative frequencies of the 10 family-types are calculated for 5000 generations, and increases or decreases in family-type frequency are 
evaluated. Because individuals carrying $A$ receive benefits from parents and grandparents who carry $A$, but at the same time receive the cost of high levels of care for the elderly, the population may reach equilibrium, when the benefit and the cost are balanced. Because those conditions cannot be solved analytically, whether the population has reached equilibrium or not is judged from population trajectories of changes in family-type frequency.

Numerical values used for the cost and benefit in the Type II model are made as consistent as possible with those values used in the age-structured population model described above. That is, based on $r$ which is subjected to a given cost and benefit, the difference in relative fitness (selection coefficient) is calculated, and numerical values for $g$ and $c$ are determined to be approximately consistent with those selection coefficients. The $p$ value (contribution from one parent carrying $A$ ) is determined, based on the basic vital statistics of the high care genotype $A a$ (i.e., before experiencing the cost and benefits) and the relatively indifferent genotype $a a$. Based on $r$ of $a a$, the $p$ value is determined as 0.15 , which is a slightly more conservative than the actual value.

\subsubsection{Spread of a High-Care Gene}

Table 4 summarizes the results obtained using the Type II discrete-generation model, showing the effects of the cost and benefit from the elderly on the evolution of care for old parents. Although these results are generally consistent with the results obtained using the aforementioned age-structured population model, the following points might be of interest.

Table 4 The effects of the costs and benefits from care for the elderly on family-type frequencies with the Type II discrete-generation model.

\begin{tabular}{|c|c|c|c|c|c|c|c|c|c|c|c|}
\hline \multirow[b]{2}{*}{$\left.\operatorname{Cost}^{2}\right)$} & \multirow[b]{2}{*}{$\mathrm{Help}^{2)}$} & \multicolumn{10}{|c|}{ Family-type Frequency ${ }^{1)}$} \\
\hline & & $\begin{array}{c}A A \underset{ }{\times} \underset{\mid}{ } \\
\\
A A\end{array}$ & $\begin{array}{c}A A \underset{ }{ } \underset{ }{\mid} \\
\\
A A\end{array}$ & $\begin{array}{c}A a \underset{1}{ } \times A a \\
\\
A A\end{array}$ & $\begin{array}{c}A A \times A a \\
\quad \times \\
A a\end{array}$ & $\begin{array}{c}A A \times a a \\
\mid \\
A a\end{array}$ & $\begin{array}{c}A a \underset{ }{\mid} \times A a \\
\quad \\
A a\end{array}$ & $\begin{array}{c}A a \times a a \\
\quad \times \\
A a\end{array}$ & $\begin{array}{c}A a \underset{ }{\mid} \times A a \\
\quad \\
a a\end{array}$ & $\begin{array}{c}A a \underset{ }{\mid} \times a a \\
\mid \\
a a\end{array}$ & $\begin{array}{c}a a \times a a \\
\mid \\
a a\end{array}$ \\
\hline \multicolumn{2}{|c|}{ initial frequency $^{3 \text { ) }}$} & 0.00000 & 0.00000 & 0.00000 & 0.00000 & 0.00000 & 0.00000 & 0.00020 & 0.00000 & 0.00020 & 0.99960 \\
\hline \multirow{6}{*}{$0.1^{4)}$} & 0.00 & 0.84157 & 0.07417 & 0.00163 & 0.07417 & 0.00325 & 0.00327 & 0.00014 & 0.00163 & 0.00014 & 0.00000 \\
\hline & 0.01 & 0.93129 & 0.03344 & 0.00030 & 0.03344 & 0.00060 & 0.00060 & 0.00001 & 0.00030 & 0.00001 & 0.00000 \\
\hline & 0.05 & 0.97249 & 0.01361 & 0.00005 & 0.01361 & 0.00010 & 0.00010 & 0.00000 & 0.00005 & 0.00000 & 0.00000 \\
\hline & 0.10 & 0.98042 & 0.00972 & 0.00002 & 0.00972 & 0.00005 & 0.00005 & 0.00000 & 0.00002 & 0.00000 & 0.00000 \\
\hline & 0.15 & 0.98422 & 0.00784 & 0.00002 & 0.00784 & 0.00003 & 0.00003 & 0.00000 & 0.00002 & 0.00000 & 0.00000 \\
\hline & 0.20 & 0.98645 & 0.00674 & 0.00001 & 0.00674 & 0.00002 & 0.00002 & 0.00000 & 0.00001 & 0.00000 & 0.00000 \\
\hline \multicolumn{2}{|c|}{ initial frequency ${ }^{3)}$} & 0.00000 & 0.00000 & 0.00000 & 0.00000 & 0.00000 & 0.00000 & 0.00020 & 0.00000 & 0.00020 & 0.99960 \\
\hline \multirow{6}{*}{$0.15^{5)}$} & 0.00 & 0.00000 & 0.00000 & 0.00000 & 0.00000 & 0.00000 & 0.00000 & 0.00000 & 0.00000 & 0.00000 & 1.00000 \\
\hline & 0.01 & 0.00000 & 0.00000 & 0.00000 & 0.00000 & 0.00000 & 0.00000 & 0.00000 & 0.00000 & 0.00000 & 1.00000 \\
\hline & 0.02 & 0.00005 & 0.00105 & 0.00579 & 0.00105 & 0.01196 & 0.01158 & 0.13101 & 0.00579 & 0.13101 & 0.70070 \\
\hline & 0.10 & 0.03886 & 0.09563 & 0.05872 & 0.09563 & 0.12727 & 0.11745 & 0.15529 & 0.05872 & 0.15529 & 0.09713 \\
\hline & 0.15 & 0.11191 & 0.16016 & 0.05721 & 0.16016 & 0.12623 & 0.11441 & 0.08967 & 0.05721 & 0.08967 & 0.03339 \\
\hline & 0.20 & 0.23502 & 0.20169 & 0.04322 & 0.20169 & 0.09674 & 0.08643 & 0.04129 & 0.04322 & 0.04129 & 0.00942 \\
\hline \multicolumn{2}{|c|}{ initial frequency ${ }^{3)}$} & 0.00000 & 0.00000 & 0.00000 & 0.00000 & 0.00000 & 0.00000 & 0.00020 & 0.00000 & 0.00020 & 0.99960 \\
\hline \multirow{7}{*}{$0.2^{5)}$} & 0.00 & 0.00000 & 0.00000 & 0.00000 & 0.00000 & 0.00000 & 0.00000 & 0.00000 & 0.00000 & 0.00000 & 1.00000 \\
\hline & 0.01 & 0.00000 & 0.00000 & 0.00000 & 0.00000 & 0.00000 & 0.00000 & 0.00000 & 0.00000 & 0.00000 & 1.00000 \\
\hline & 0.02 & 0.00000 & 0.00000 & 0.00000 & 0.00000 & 0.00000 & 0.00000 & 0.00000 & 0.00000 & 0.00000 & 1.00000 \\
\hline & 0.05 & 0.00000 & 0.00000 & 0.00000 & 0.00000 & 0.00000 & 0.00000 & 0.00000 & 0.00000 & 0.00000 & 1.00000 \\
\hline & 0.10 & 0.00002 & 0.00061 & 0.00405 & 0.00061 & 0.00890 & 0.00811 & 0.11579 & 0.00405 & 0.11579 & 0.74206 \\
\hline & 0.15 & 0.00063 & 0.00657 & 0.01697 & 0.00657 & 0.03809 & 0.03394 & 0.19338 & 0.01697 & 0.19338 & 0.49349 \\
\hline & 0.20 & 0.00262 & 0.01746 & 0.02883 & 0.01746 & 0.06588 & 0.05765 & 0.21354 & 0.02883 & 0.21354 & 0.35419 \\
\hline
\end{tabular}

(a) If the cost of elderly care is sufficiently small, the behavior of care for old parents may increase in frequency up to near fixation within the population, even without the benefit of the help from the elderly.

(b) If the elderly only impose a heavy cost of elderly care on younger generations without providing any benefits from helping, the behavior of care for old parents may be eliminated from the population (because of the assumed pleiotropic 
World Journal of Advanced Research and Reviews, 2020, 08(02), 189-202

constraints, high levels of care for infants are also eliminated). However, if a sufficient amount of help is provided by the elderly, the behavior of care for old parents could increase within the population despite a heavy cost of elderly care.

(c) If the cost of elderly care is high, the frequencies of the high care family-type $(A A \times A A-A A)$ may increase but reach equilibrium at intermediate levels, even with benefits of help from the elderly. The larger the benefits from the elderly, the higher the equilibrium frequencies of the high care family-type.

Similarly to previous models [1], the results obtained using the Type II discrete-generation model suggest that a high level of benefits of help by the elderly, and as low a cost of care for the elderly as possible, are necessary not only for maintaining the behavior of care for old parents as well as high levels of care for infants, but also for enhancing the rate of population growth.

\section{Discussion}

The aim of this study is to consider how the behavior of care for the elderly could evolve within a population, in which population aging coupled with a tendency toward a decrease in the number of younger generations is progressing steadily as in such countries like Japan. To be sure, Japan is representative of countries suffering from population aging coupled with a decrease in the number of children. Although I indeed use population statistics of Japan in my study [1], my intention is to create a hypothetical population suffering from these demographic difficulties, not to project precisely the fate of the Japanese population in the future. Therefore, it should be noted that a lot of details concerning the Japanese population, which are not directly relevant to the present study, can be ignored here.

In this study, I use the dichotomous distinction in care for old parents as well as infants, high care vs relatively indifferent, as in Miyo [1]. As mentioned in the text, I do not envisage any specific form of behavior for each category, and use these terms in the same way as altruistic and non-altruistic in the literature concerning the evolution of altruistic behavior. However, it seems probable that there might be some continuous variations in this behavior from filial piety at one extreme to some tragic forms of behavior such as elderly and/or child maltreatment at the other extreme. Therefore, representing this widely varying behavior dichotomously as high care and relatively indifferent is done just for simplicity.

In this study, using some evolutionary genetic models assuming pleiotropic constraints between care for old parents and care for infants, I attempt to consider how care for old parents could evolve within the population, in which several demographic difficulties, including population aging and diminishing population size, are in progress. It is suggested that high levels of care for old parents could evolve within the population if the cost of elderly care is not too heavy, but it is also suggested that, if care of the elderly imposes an enormous cost on younger generations, this behavior might be eliminated from the population, resulting in the exclusion of high levels of care for infants as well. Although little research has so far been conducted on the evolutionary consequences of elderly care for population dynamics, it may be important to pay attention to the current situations within the population, where population aging and a decrease in population size are progressing steadily. For example, in Japan, child maltreatment and elderly maltreatment are getting more and more serious during recent years. Figure 5 illustrates a recent tendency in Japan toward an increase in the number of persons arrested for child maltreatment by the police, as well as the number of cases involving the elderly maltreated by family caregivers, judged by municipalities. Although many factors, social, economic and biological, may contribute to this situation [27], in the light of findings obtained in this study, this tendency could be looked on as the process, in which the frequency of the relatively indifferent allele is increasing within the population.

In this study, the evolutionary biology of care for the elderly is considered using some models with a single locus with two alleles, high care and relatively indifferent. If the cost of elderly care is too high, a relatively indifferent allele may indeed invade the population; for instance, Figure 6 indicates a result obtained using the Type II model, where $p=0.15$, $g=0$ and $2 c=0.30$. Although the real situations may not be as simple as in the models used in this study, social situations in which the relatively indifferent genotype is more advantageous than the high care genotypes, could be realized in the future, if care of the elderly imposes an enormous cost on younger generations.

Many studies have demonstrated the effects of interactions between genotypic variation in the monoamine oxidase A (MAOA) activity levels and social experiences, including child maltreatment and provocation, on aggressive and/or antisocial behaviors [27-30]. Although it is not implied here that the relatively indifferent allele is the same as in a gene such as MAOA-L (low activity allele of MAOA), situations in which the frequency of the relatively indifferent gene can increase may approximate the type of social adversity in which genes like MAOA-L could affect human behavior in aggressive and/or antisocial manners. Therefore, the situation illustrated in Figure 5 may signal serious social adversity in the near future. 


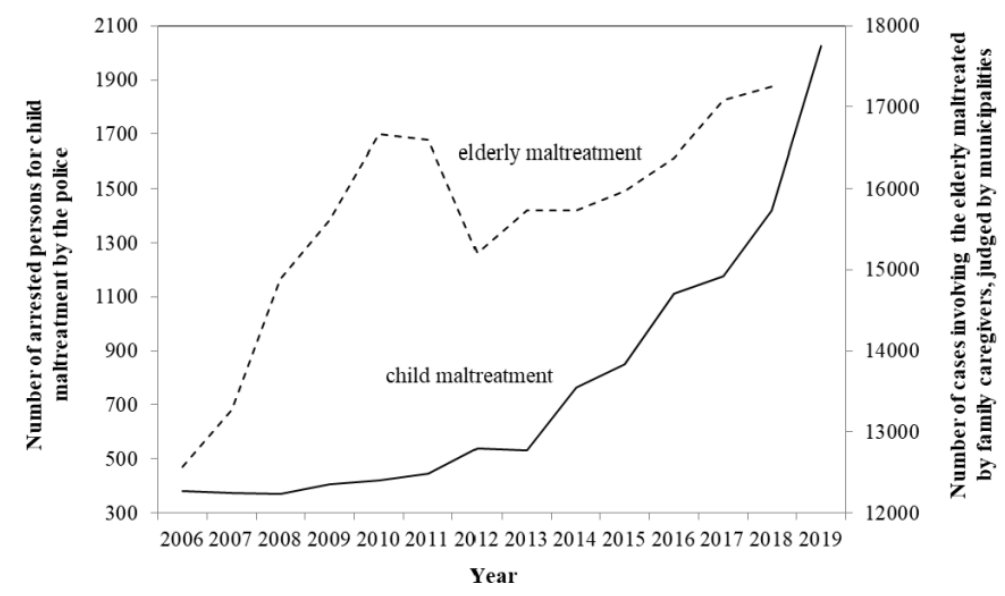

Figure 5 Changes in the number of arrested persons for child maltreatment by the police, and in the number of cases involving the elderly maltreated by family caregivers, judged by municipalities, in Japan.

[Sources: data for child maltreatment, released by National Police Agency (Japan), available at. https://www.npa.go.jp/safetylife/syonen/hikou_gyakutai_sakusyu/R1.pdf (in Japanese; accessed 26 June 2020), and data for elderly maltreatment, released by Ministry of Health, Labour and Welfare (Japan) available at https://www.mhlw.go.jp/content/12304250/000584234.pdf (in Japanese; accessed 26 June 2020)]

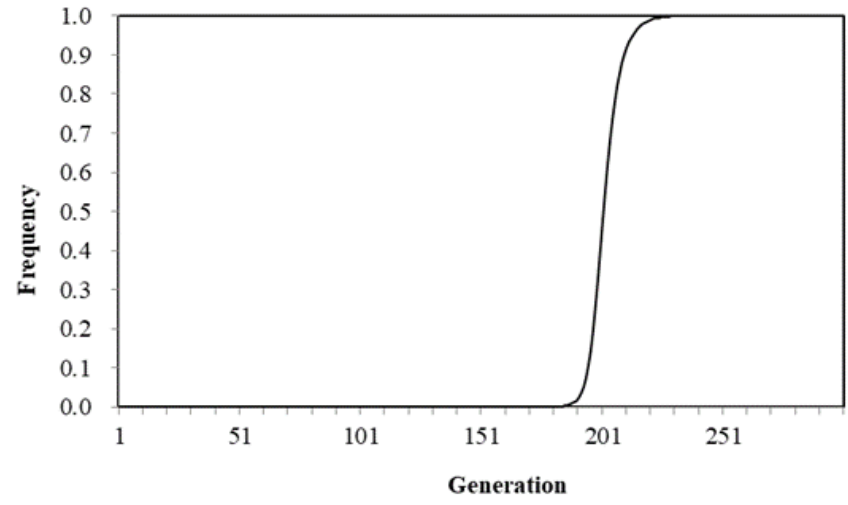

Figure 6 Increase of a relatively indifferent allele within the population, a majority of which has the high care genotype, with Type II model. The initial frequency of this allele is 0.01 . The values of $p, g$, and $2 c$ are $0.15,0$, and 0.30 , respectively. The situation, in which only a heavy cost is imposed on younger generations without the benefit of the help from the elderly, is envisaged.

In this study, it is suggested that the benefit of the help from elderly people is essential not only for the behavior of care for old parents to be maintained within the population, but also for the population to increase, even though there may be a high cost of care for old parents. If this is the case, would conditions in which as high a benefit of help can be derived from the elderly as possible have been realized in countries where population aging is progressing gradually? In Japan, for example, the percentage of women aged over 65 years living alone (one-person households) is estimated to keep on increasing, and in 2040, about one out of four elderly women aged over 65 years are expected to live alone [31]. Elderly people in such countries like Japan may be in a situation where they are not capable of contributing to their children and grandchildren, even though they may very much wish to make contributions to younger generations. If we receive only a high cost of elderly care without the benefit of the help effects from the elderly, the behavior of care for the elderly as well as high levels of care for infants may be eliminated from the population, the population may diminish further, and social situations may get worse.

According to the grandmother hypothesis, a prolonged post-reproductive lifespan of the elderly will be favored by selection, if this can increase their inclusive fitnesses through making contributions to reproductive success of their own offspring [9]. Broadly speaking, therefore, in species with extensive social interactions between different generations the elderly, especially grandmothers, may have evolved to make fitness contributions to their successors. 
In this evolutionary process, grandmothering may have had consequences for human aging, which at present raises some serious concerns in the human populations, for which delayed childbearing and isolated nuclear families are characteristic [32]. For example, occupants of nursing homes for the elderly with dementia, especially women suffering from this disease, usually keep their abilities to do housekeeping work, such as cooking, cleaning and so on (the author's care-worker and care-manager experiences). In addition, Danielsbacka et al. [33] found associations between grandparenting and health and well-being of the elderly. Therefore, the current situations the elderly are put in, where they cannot contribute to their younger generations, may be different from the social environments to which human beings have been adapted for a long time, and may be pathological for the elderly. Although dementia is a serious matter at present and will be more serious in the near future, viewing it from the standpoint of the environment of evolutionary adaptedness [34] may provide clues for better care in the future. In addition, various theories have been presented concerning how natural selection could affect Alzheimer's disease, and the possibility that Alzheimer's is a "disease of civilization" has been proposed [35]. Findings from the perspective on evolutionary medicine may indeed provide helpful and useful insights into the realities of care-working. A variety of perspectives on current situations that are filled with a lot of difficulties is essential for the future.

A foundation for the models which I have used so far is the assumption that there are pleiotropic constraints between high levels of care for old parents and high levels of care for infants. At this point, there should be no doubt (a) that it is absolutely impossible for us to survive without care from others (usually parents) during infancy [16, 17], so that establishing a solid relationship with others (usually parents) should be strongly advantageous, compared with the case where such relationships cannot be established, (b) that care of the elderly imposes a large cost on us, including money, energy, time, and so on, and nevertheless (c) that most people, as long as I know, do not abandon their old parents but take care of them, even though the elderly have ceased reproduction (that is, in terms of inclusive fitness, care of the elderly provides no benefit but only a cost, so that it is surprising that this behavior has not been eliminated from the population).

Based on these facts, I have used evolutionary genetic models, assuming pleiotropic constraints between care for old parents and care for infants, in order to consider how care of the elderly could evolve in the future within a population where population aging, coupled with a tendency toward a decrease in population size, is in steady progress. Although it is desirable for the relevance of the models used so far to be carefully examined, it is anticipated in such countries like Japan that more harsh situations would be brought about in the near future [5], so that we may not have enough time to examine these models seriously. However, 50 or 100 years later, by inspecting the societies where population aging coupled with a decrease in population size is anticipated to have progressed to a higher level (such as Japan), it might be possible to evaluate the relevance of the models presented here. In this sense, we are going to experience precisely how we may evolve in the course filled with many demographic difficulties described above.

\section{Conclusion}

In conclusion, using some evolutionary genetic models that assume pleiotropic constraints among care for old parents and care for infants, it is suggested that the behavior of care for the elderly as well as high levels of care for infants may be eliminated from the population, the population may diminish further, and social situations may get worse, if only an enormous cost of elderly care is imposed on younger generations without the benefit of the help effects from the elderly. Therefore, we should consider how we could derive the help effects from the elderly as much as possible and how we could manage an enormous cost of elderly care as neatly as possible, in order not only for the behavior of care for old parents as well as high levels of care for infants to be maintained within the population, but also for the population to be increased even with a heavy cost of elderly care.

\section{Compliance with ethical standards}

\section{Acknowledgments}

I would like to express my sincere appreciation to Professor Brian Charlesworth at the University of Edinburgh for his critical comments and valuable suggestions on the manuscript.

\section{Disclosure of conflict of interest}

I declare no conflict of interest. 


\section{References}

[1] Miyo T. Why do we care for old parents? Evolutionary genetic model of elderly caring. Open Journal of Genetics. 2017; 7: 20-39.

[2] Hamilton WD. The evolution of altruistic behavior. American Naturalist. 1963; 97: 354-356.

[3] Hamilton WD. The genetical evolution of social behaviour, I. Journal of Theoretical Biology. 1964; 7: 1-16.

[4] Hamilton WD. The genetical evolution of social behaviour, II. Journal of Theoretical Biology. $1964 ;$ 7: 17-52.

[5] Cabinet Office Japan. Annual report on the aging society [Summary]. 2017.

[6] Charlesworth B. Evolution in age-structured populations, 2nd ed. Cambridge, UK: Cambridge University Press; 1994.

[7] Lahdenperä M, Lummaa V, Helle S, Tremblay M, Russell AF. Fitness benefits of prolonged post-reproductive lifespan in women. Nature. 2004; 428: 178-181.

[8] Nattrass S, Croft DP, Ellis S, Cant MA, Weiss MN, Wright BM, Stredulinsky E, Doniol-Valcroze T, Ford JKB, Balcomb KC, Franks DW. Postreproductive killer whale grandmothers improve the survival of their grandoffspring. Proceedings of the National Academy of Sciences of the United States of America. 2019; 116: 26669-26673.

[9] Hawkes K, O'Connell JF, Blurton Jones NG, Alvarez H, Charnov EL. The grandmother hypothesis and human evolution. In: Cronk L, Chagnon N, Irons W, eds. Adaptation and Human Behavior: an Anthropological Perspective. New York, USA: Routledge; 2000. p. 237-258.

[10] Hawkes K. The grandmother effect. Nature. 2004; 428: 128-129.

[11] Kim PS, Coxworth JE, Hawkes K. Increased longevity evolves from grandmothering. Proceedings of the Royal Society B. 2012; 279: 4880-4884.

[12] Lahdenperä M, Gillespie DOS, Lummaa V, Russell AF. Severe intergenerational reproductive conflict and the evolution of menopause. Ecology Letters. 2012; 15: 1283-1290.

[13] Chapman SN, Pettay JE, Lummaa V, Lahdenperä M. Limits to fitness benefits of prolonged post-reproductive lifespan in women. Current Biology. 2019; 29: 645-650.

[14] Crow JF, Aoki K. Group selection for a polygenic behavioral trait: a differential proliferation model. Proceedings of the National Academy of Sciences of the United States of America. 1982; 79: 2628-2631.

[15] Lamb ME, Thompson RA, Gardner W, Charnov EL. Infant-mother attachment: the origins and developmental significance of individual differences in strange situation behavior. Hillsdale, New Jersey, USA: Lawrence Erlbaum Associates; 1985. p. 45-58.

[16] Bowlby J. Psychoanalysis as a natural science. International Review of Psycho-Analysis. 1981; 8: 243-256.

[17] Hrdy SB. Mothers and others: the evolutionary origins of mutual understanding. Cambridge, Massachusetts, USA: The Belknap Press of Harvard University Press; 2009. p. 209-232.

[18] Arnhart L. Darwinian natural right: the biological ethics of human nature. New York, USA: State University of New York Press; 1998. p. 89-121.

[19] Charlesworth B, Charlesworth D. Elements of evolutionary genetics. Greenwood Village, Colorado, USA: Roberts and Company Publishers; 2010. p. 47-142.

[20] Kaplan H. The evolution of the human life course. In: Wachter KW, Finch CE, eds. Between Zeus and the Salmon: the Biodemography of Longevity. Washington DC, USA: National Academy Press; 1997. p. 175-211.

[21] Hawkes K. Grandmothers and the evolution of human longevity. American Journal of Human Biology. 2003; 15: 380-400.

[22] Charlesworth B, Charnov EL. Kin selection in age-structured populations. Journal of Theoretical Biolog. 1981; 88: 103-119.

[23] Charlesworth B. Some models of the evolution of altruistic behaviour between siblings. Journal of Theoretical Biology. 1978; 72: 297-319.

[24] Charlesworth B, Charlesworth D. A model for the evolution of dioecy and gynodioecy. American Naturalist. 1978; 112: 975-997. 
[25] Beise J. The helping and the helpful grandmother: the role of maternal and paternal grandmothers in child mortality in the seventeenth- and eighteenth-century population of French settlers in Québec, Canada. In: Voland E, Chasiotis A, Schiefenhövel W, eds. Grandmotherhood: the Evolutionary Significance of the Second Half of Female Life. New Brunswick, New Jersey, USA: Rutgers University Press; 2005. p. 215-238.

[26] Lamb ME, Pleck JH, Charnov EL, Levine JA. A biosocial perspective on paternal behavior and involvement. In: Lancaster JB, Altmann J, Rossi AS, Sherrod LR, eds. Parenting Across the Life Span: Biosocial Dimensions. New York, USA: Routledge; 1987. p. 111-142.

[27] Frazzetto G, Di Lorenzo G, Carola V, Proietti L, Sokolowska E, Siracusano A, Gross C, Troisi A. Early trauma and increased risk for physical aggression during adulthood: the moderating role of MAOA genotype. PLoS ONE. 2007; 2(5): e486.

[28] Caspi A, McClay J, Moffitt TE, Mill J, Martin J, Craig IW, Taylor A, Poulton R. Role of genotype in the cycle of violence in maltreated children. Science. 2002; 297: 851-854.

[29] McDermott R, Tingley D, Cowden J, Frazzetto G, Johnson DDP. Monoamine oxidase A gene (MAOA) predicts behavioral aggression following provocation. Proceedings of the National Academy of Sciences of the United States of America. 2009; 106(7): 2118-2123.

[30] Rehan W, Sandnabba NK, Johansson A, Westberg L, Santtila P. Effects of MAOA genotype and childhood experiences of physical and emotional abuse on aggressive behavior in adulthood. Nordic Psychology. 2015; 67(4): 301-312.

[31] Cabinet Office Japan. Annual report on the aging society [in Japanese]. 2019.

[32] Hawkes K. How grandmother effects plus individual variation in frailty shape fertility and mortality: guidance from human-chimpanzee comparisons. Proceedings of the National Academy of Sciences of the United States of America. 2010; 107: 8977-8984.

[33] Danielsbacka M, Tanskanen AO, Coall DA, Jokela M. Grandparental childcare, health and well-being in Europe: a within-individual investigation of longitudinal data. Social Science and Medicine. 2019; 230: 194-203.

[34] Stevens A, Price J. Evolutionary psychiatry: a new beginning, 2nd ed. London, UK: Routledge; 2000.

[35] Fox M. 'Evolutionary medicine' perspectives on Alzheimer's disease: review and new directions. Ageing Research Reviews. 2018; 47: 140-148. 